\title{
NONTANGENTIAL INTERPOLATING SEQUENCES AND INTERPOLATION BY NORMAL FUNCTIONS
}

\section{KAM-FOOK TSE}

Abstract. The first part of the paper shows that a sequence of points in the unit disk of the complex plane, tending nontangentially to a point on the unit circle, is an interpolating sequence if and only if the pseudo-hyperbolic distance between any pair of points in the sequence is bounded away from zero. The second part shows that interpolating sequences for bounded analytic functions are also interpolating sequences for normal functions.

1. Introduction. Let $D=\{|z|<1\}$ and $C=\{|z|=1\}$. For $z, z^{\prime} \in D$, let

$$
\phi\left(z, z^{\prime}\right)=\left(z-z^{\prime}\right) /\left(1-\bar{z}^{\prime} z\right)
$$

and let $\psi\left(z, z^{\prime}\right)=\left|\phi\left(z, z^{\prime}\right)\right|$ denote the pseudo-hyperbolic distance between $z$ and $z^{\prime}$. If $S$ and $S^{\prime}$ are two disjoint subsets in $D$, then $\psi\left(S, S^{\prime}\right)=\inf \left\{\psi\left(z, z^{\prime}\right): z \in S^{\prime}, z^{\prime} \in S^{\prime}\right\}$. As usual, $H^{\infty}$ and $l^{\infty}$ will denote the space of bounded analytic functions in $D$ and the space of bounded sequences of complex numbers, respectively. Finally, a sequence $\left\{z_{n}\right\}$ in $D$ is called an interpolating sequence (or $\left\{z_{n}\right\}$ interpolates) if for each $\left\{w_{n}\right\} \in l^{\infty}$, there exists $f \in H^{\infty}$ such that $f\left(z_{n}\right)=w_{n}$ for $n$ $=1,2, \cdots$. A criterion that $\left\{z_{n}\right\}$ interpolates is given by L. Carleson $[1]$ as

$$
A(n)=\prod_{j=1 ; j \neq n}^{\infty} \psi\left(z_{j}, z_{n}\right) \geqq \delta>0
$$

for $n=1,2, \cdots$. Intuitively, the Carleson's condition indicates that the points in $\left\{z_{n}\right\}$ should not "lie too close" to one another, in the pseudo-hyperbolic metric $\psi$, in order to be an interpolating sequence. On the other hand, it is apparent that

$$
\psi\left(z_{n}, z_{m}\right) \geqq \delta>0
$$

for all $m \neq n$, is a necessary condition for $\left\{z_{n}\right\}$ to be an interpolating sequence. However, a more general criterion in terms of pseudohyperbolic metric to replace $(C)$ is not easy to find. Newman in $[4$, Theorem 3, p. 506] had obtained the following result along this direction for a particular case.

Received by the editors August 10, 1970.

AMS 1970 subject classifications. Primary 30A74, 30A80; Secondary 30A76.

Key words and phrases. Pseudo-hyperbolic distance, interpolating sequence, Blaschke product, Blaschke sequence, normal function. 
THEOREM N. Let $\left\{z_{n}\right\}$ be a sequence of points in $D$ such that it converges to +1 in a nontangential manner. Then $\left\{z_{n}\right\}$ is an interpolating sequence if and only if the set

$$
\left\{\frac{1-z_{m}}{1-z_{n}}: m \neq n\right\}
$$

does not have +1 as a limit point.

In $\$ 2$, we shall show a version of Theorem $\mathrm{N}$ in terms of pseudohyperbolic metric. And we shall consider the extension of the interpolating problem by functions in $H^{\infty}$ to that by normal functions and functions of bounded type in $\S 3$.

2. Interpolating sequences in a Stolz angle. A Blaschke sequence $S$ in $D$ is a sequence of points $S=\left\{z_{n}\right\} \subset D$ with $\sum\left(1-\left|z_{n}\right|\right)<\infty$. The Blaschke product associated with such a Blaschke sequence is the well-defined infinite product $\prod_{n=1}^{\infty}\left(z_{n} /\left|z_{n}\right|\right) \phi\left(z, z_{n}\right)$. It is evident that every interpolating sequence is also a Blaschke sequence.

THEOREM 1. If $\left\{z_{n}\right\}$ is a sequence of points contained in a Stolz angle in $D$, then $\left\{z_{n}\right\}$ interpolates if and only if $\psi\left(z_{m}, z_{n}\right) \geqq \delta>0$ for all $m \neq n$.

Proof. The necessity can be proved by (1) and we shall show the sufficiency. Suppose that the Stolz angle has its vertex at 1. By Theorem $N$, if $\left\{z_{n}\right\}$ does not interpolate, there are sequences $\left\{m_{k}\right\}$ and $\left\{n_{k}\right\}, m_{k} \neq n_{k}$, such that

$$
\left(1-z_{m_{k}}\right) /\left(1-z_{n_{k}}\right) \rightarrow 1 \text { and }\left(1-z_{m_{k}}\right) /\left(1-\bar{z}_{n_{k}}\right) \rightarrow \theta,
$$

$|\theta|=1, \theta \neq-1$. Suppressing the subscript $k$ and using the equalities

$$
z_{n}-z_{m}=\left(1-z_{m}\right)-\left(1-z_{n}\right)
$$

and

$$
1-\bar{z}_{n} z_{m}=\left(1-z_{m}\right)+z_{m}\left(1-\bar{z}_{n}\right)
$$

we obtain

$$
\frac{z_{n}-z_{m}}{1-\bar{z}_{n} z_{m}}=\frac{\left(1-z_{m}\right)-\left(1-z_{n}\right)}{1-z_{m}} \cdot \frac{1-z_{m}}{\left(1-z_{m}\right)+z_{m}\left(1-\bar{z}_{n}\right)}
$$

where the first factor on the right of (4) tends to 0 and the second to $1 /(1+\theta)$. Hence we obtain $\inf \left\{\psi\left(z_{n}, z_{m}\right): m \neq n\right\}=0$. Since $\psi\left(z_{n}, z_{m}\right)$ is invariant under a rotation, the condition is sufficient at each point of the unit circle. 
3. Interpolation by normal functions. In this section, we shall show that interpolating sequences for bounded analytic functions are also interpolating sequences for normal functions. We have not seen any results on either approximation or interpolation by normal functions. As before, we let $B(z, S)$ be the Blaschke product associated with the Blaschke sequence $S$ in $D$.

Lемма 3. Let $S=\left\{z_{1}, z_{2}, \cdots\right\}$ be an interpolating sequence in $D$ and $\left\{w_{0}, w_{1}, w_{2}, \cdots\right\} \in l^{\infty}$. Then for any $\epsilon>0$ and $\eta>0$, there exists an $f \in H^{\infty}$ such that $f\left(z_{n}\right)=w_{n}$ and $\left|f(z)-w_{0}\right| \geqq \eta$, whenever $\psi(z, S) \geqq \epsilon$.

Proof. Let $w_{0}=0$, for otherwise, we may let $g(z)$ interpolate $\left\{w_{n}^{\prime}=w_{n}-w_{0}\right\}$ and let $f(z)=g(z)+w_{0}$. Since $S$ interpolates, there exists a $k \in H^{\infty}$ such that $k\left(z_{n}\right)=w_{n}$ for $n=1,2, \cdots$. Moreover, let $k$ be bounded by $K$. By [2, Theorem 1$]$, there exists a $\delta>0$ such that $|B(z, S)| \geqq \delta$ whenever $\psi(z, S) \geqq \epsilon$. Now, $f(z)=k(z)+((K+\eta) / \delta)$ $\cdot B(z, S)$ will be the required function.

TheоRем 2. If $S=\left\{z_{p}\right\}$ is an interpolating sequence in $D$ and $\left\{w_{p}\right\}$ is any sequence of points on the Riemann sphere $\Omega$, then there exists a function $H(z)$ in $D$, which is normal and is of bounded type such that $H\left(z_{p}\right)=w_{p}$ for $p=1,2, \cdots$.

Proof. Since $S$ interpolates, we may let $\psi\left(z_{m}, z_{n}\right) \geqq \delta>0$ for all $m \neq n$. Let $S_{1}=\left\{z_{n} \in S:\left|w_{n}\right| \leqq 1\right\}$ and $S_{2}=\left\{z_{n} \in S:\left|w_{n}\right|>1\right\}$. $\left(S=S_{1} \cup S_{2}\right.$.) We shall construct two functions $F(z)$ and $G(z)$ in $H^{\infty}$ such that

(a) $F\left(z_{n}\right)=w_{n}$ for $z_{n} \in S_{1}$;

(b) $|F(z)| \geqq \eta>0$ for $z \in D-U$, where $U=\left\{z: \psi\left(z, S_{1}\right)<\xi\right\}$, for some $\xi>0$ which is to be determined later;

(c) $G\left(z_{n}\right)=1$ for $z_{n} \in S_{1}$ and $G\left(z_{n}\right)=F\left(z_{n}\right) / w_{n}$ for $z_{n} \in S_{2}$ (if $w_{n}=\infty$, let $G\left(z_{n}\right)=0$ ) ;

(d) $|G(z)| \geqq \frac{1}{2}$ for $z \in U$.

If we let $H(z)=F(z) / G(z)$, then it is evident that

(a') $H\left(z_{p}\right)=w_{p}$ for $p=1,2, \cdots$,

(b') $H(z)$ is of bounded type, and we shall show

(c') $H(z)$ is normal in $D$.

If it were not the case, then by [5, Corollary 1.2, p. 64] there exists $S_{z}=\left\{z_{q}\right\} \subset D$ with $\lim F\left(z_{q}\right)=0$ and $\lim G\left(z_{q}\right)=0$. By (b), $S_{3} \subset U$, but by (d), $S_{3} \subset D-U$. Hence $H(z)$ is normal in $D$.

Now we begin to construct $F(z)$ and $G(z)$. By Lemma 3, there exists an $F(z) \in H^{\infty}$ such that conditions (a) and (b) hold. On the other hand, there exist $f, g \in H^{\infty}$ such that 
(5) $f\left(z_{n}\right)=0, \quad$ for $z_{n} \in S_{1}, \quad f\left(z_{n}\right)=1, \quad$ for $z_{n} \in S_{2}$,

(6) $g\left(z_{n}\right)=0, \quad$ for $z_{n} \in S_{1}, \quad g\left(z_{n}\right)=F\left(z_{n}\right) / w_{n}-1$, for $z_{n} \in S_{2}$.

Moreover, let $g(z)$ be bounded by $M$ for some $M>0$. Since $f(z)$ is normal in $D$, there exists a $\xi$, such that $0<\xi<\delta$ and $|f(z)|<\frac{1}{2}$ for $z \in U$ (see [3]). Choose an integer $N>0$ such that $M<2^{N-1}$ and let

$$
h(z)=(f(z))^{N} g(z) .
$$

Note that for $z \in U,|h(z)| \leqq\left(\frac{1}{2}\right)^{N} \cdot M<\frac{1}{2}$. Finally, we let $G(z)=1$ $+h(z)$. It is easy to see from (5), (6) and (7) that $G\left(z_{n}\right)=1$ for $z_{n} \in S_{1}$, $G\left(z_{n}\right)=F\left(z_{n}\right) / w_{n}$ for $z_{n} \in S_{2}$. Moreover, we have in $U$,

$$
|G(z)| \geqq 1-|1-G(z)|=1-|h(z)| \geqq 1-\frac{1}{2}=\frac{1}{2} ;
$$

i.e., $G(z)$ satisfied conditions (c) and (d). Hence our proof is complete.

CoROllary 1. If $S=\left\{z_{n}\right\}$ is a sequence of points contained in a Stolz angle in $D$, then $S$ is an interpolating sequence for normal functions if and only if $\psi\left(z_{m}, z_{n}\right) \geqq \delta>0$ for all $m \neq n$.

\section{REFERENCES}

1. L. Carleson, An interpolation problem for bounded analytic functions, Amer. J. Math. 80 (1958), 921-930. MR 22 \#8129.

2. J. A. Cima and P. Colwell, Blaschke quotients and normality, Proc. Amer. Math. Soc. 19 (1968), 796-798. MR 37 \#3007.

3. P. Lappan, Some sequential properties of normal and non-normal functions with applications to automorphic functions, Comm. Math. Univ. St. Paul. 12 (1964), 41-57. MR 29 \#250.

4. D. J. Newman, Interpolation in $H^{\infty}$, Trans. Amer. Math. Soc. 92 (1959), 501507. MR $22 \# 8130$.

5. K. F. Tse, On the sums and products of normal functions, Comm. Math. Univ. St. Paul. 17 (1969), 63-72.

Syracuse University, Syracuse, New York 13210 